EPJ Web of Conferences 41, 09008 (2013)

DOI: $10.1051 /$ epjconf/20134109008

C) Owned by the authors, published by EDP Sciences, 2013

\title{
Second harmonic generation in NLO polymers excited by Surface Plasmon enhanced electric field induced by femtosecond optical pulses
}

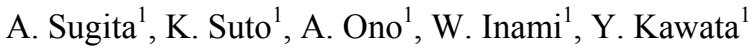 \\ ${ }^{1}$ Faculty of Engineering, Shizuoka University, Hamamatsu, Shizuoka 432-8561, Japan
}

\begin{abstract}
We will report second harmonic generation (SHG) in nonlinear optical (NLO) polymers excited by surface plasmon enhanced optical fields. The surface plasmon (SP) polariton was excited in an attenuated total reflection geometry having the Kretchmann configuration. The NLO polymers, consisting of Disperse Red1 as guest chromophores and poly (methyl methacrylate) as host materials, were coated upon the Ag layers. Our experimental results indicated that the SHG signal intensity from the polymer coated Ag films was more than 10 times higher than that from the non-coated Ag films. The SHG autocorrelation traces excited by SP-enhanced fields were also studied and the correlation time was shorter than $150 \mathrm{fs}$, the temporal resolutions of the present spectrometer.
\end{abstract}

\section{Introduction}

Great attentions have been paid for Surface Plasmon (SP) and its applications to nanophotonics in the field of ultrafast optics. The SP, known as collective motions of conduction band electrons pumped by light waves, accomanies the enhanced optical fields in the vicinity of the metal surface. The intensities of the SP-enhanced fields are typically 2-3 magnitudes higher than those of the incidence light waves. The SP-enhanced optical fields pumped by femtosecond optical pulses have attracted as novel excitation sources for nonlinear optics (NLO) because of their high peak intensities due to the spatial and temporal confinements. For examples, the intensities of the SP-enhanced fields pumped by the nJ-level 10 fs-optical pulses from mode-locked oscillators are high enough to accelerate electrons with $\sim \mathrm{kV}$ kinetic energies or to generate higher harmonics generations $[1,2]$.

On the other hand, not so much attentions seems to be paid to the SP-enhanced fields from the viewpoint of the excitation light sources for the second-order NLO, the lowest order NLO phenomena, even though a lot of studies have dealt with the second-order NLO susceptibilities of the metal surface itself where the SP was created. It was partly because of the difficulties in preparing NLO active materials with noncentrosymmetric structures on the metal surface. However, there are enoumous demands for the nonlinear optical frequency converters for the SP-enhanced optical fields. The frequency regions available for the SP enhanced fields are limited at the frequencies lower than the surface plasma frequency of the metal materials. In the present study, we examined second-order nonlinear susceptibility in NLO polymers pumped by SP-enhanced optical fields and its ultrafast response. Here, we dealt with the NLO polymer materials which self-organized noncentrosymmetric structures just by depositing on the metal surfaces [3].

This is an Open Access article distributed under the terms of the Creative Commons Attribution License 2.0, which permits unrestricted use, distribution, and reproduction in any medium, provided the original work is properly cited. 


\section{Experimental}

The SP was excited by an attenuated total reflection (ATR) geometry having Kretschmann configuration. The samples for the NLO spectroscopy consisted of three layer structures. A 40 $\mathrm{nm}$-silver thin film was coated on a BK7 glass prism, above which a thin film of NLO polymer was coated. The NLO polymers were the host-guest materials, consisting of poly (methyl methacrylate) as host and DR1 (4-[Ethyl(2hydroxyethyl)amino]-4'-nitroazobenzene) as the guest. The polymer fabricated on the metals exhibited second-order nonlinear optical susceptibility just by annealing the materials, as reported in our previous studies [3]. The thickness of the polymer layers were $15-100 \mathrm{~nm}$.

The apparatus for measuring the second order NLO

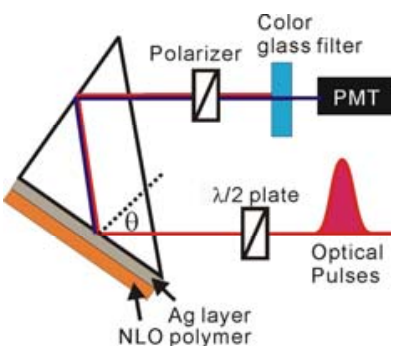

Fig. 1 Schematics of optical geometry for measuring the second harmonic generations at the SP resonance.

susceptibilities is shown in Fig. 1. The excitation light sources were the femtosecond optical pulses (center wavelength : $800 \mathrm{~nm}$, pulse width : $150 \mathrm{fs}$ and pulse energy : $1 \mathrm{~mJ}$ ). The reflected SHG signals were detected with a photomultiplier tube after separating residual fundamental lights by a color glass filter. The beam diameter on the sample surface was approximately $\phi \sim 5 \mathrm{~mm}$. The SHG signals were recorded as functions of the polarization angles of the excitation lights. The polarization dependence of the SHG signals was also resolved with an analyzer.

\section{Results and Discussion}

Fig. 2(a) is the ATR and the SHG signal profiles of the NLO polymer-coated silver films against the incidence angle of the $p$-polarized excitation lights. Here, the data are shown for the samples with two different polymer film thicknesses. The ATR and the SHG profiles of the uncoated silver film are also shown as a reference. Both of the polymer-coated and uncoated silver films have a dip structure due to the SP resonance. The minimum reflectivity in the ATR profile occurred at $\theta_{s p}=41.2$ ${ }^{\circ}$ for the non-coated $\mathrm{Ag}$ films. The position of $\theta_{s p}$ depended on the thickness of the polymer layer and the samples with wider polymer thickness exhibted the SP responance at wider angles. The width of the dip was wider for the samples with thicker polymer layers. The dip width is closely associated with the intensity of the SP-enhanced fields, and the narrower dip width is characterized by higher SP field intensity enhancements. Hence, the SP-enhanced field intensity is higher in the thinner NLO polymers. On the other hand, the SHG profiles correspond well to the ATR profiles and the intensity was the highest at $\theta_{s p}$.

The SHG signal intensities are plotted as a function of the pump light powers $I_{\text {pump }}$ in Fig. 2(b). The curves were proportinal to $I_{\text {pump }}^{2}$, like in the case of the secondorder NLO for the propagating light waves. The SHG signal intensities from the polymer-coated
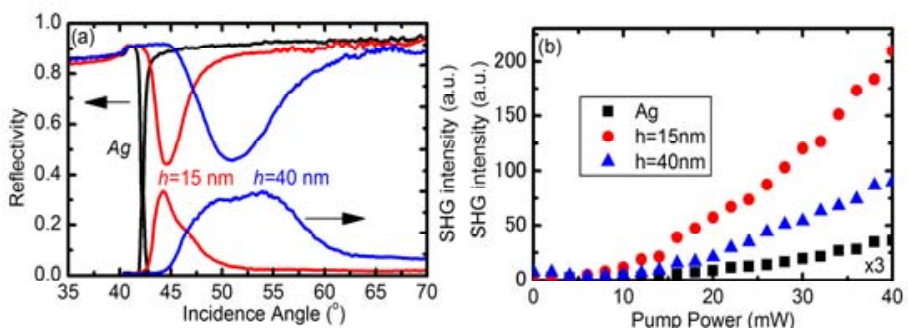

Fig. 2 (a) The ATR and SHG signal profiles against incidence angles of the pump beam for the polymer films coated on Ag layers. The same data for the Ag layer is also shown as a reference. (b) The SHG signal intensity as a function of the excitation beam intensities.

Ag films were higher than those of the uncoated Ag films. Hence, it is concluded that the SPenhanced fields excited nonlinear polarizations inside the NLO polymers as well as on the surface of the Ag films, and the effective nonlinearity of the former is higher than that of the latter. 
The SH signal intensity is plotted against the pump light polarization angles $\gamma_{\mathrm{p}}$ in the SP-resonance conditions (Fig. 3(a)). Here, the thickness of the polymer layer was $15 \mathrm{~nm}$. The curve is reproduced well with a function of $\cos ^{4} \gamma_{p}$. The result indicates that the nonlinear polarizations are preferably excited by the $p$ polarized light waves. The polarization angle $\gamma_{\mathrm{a}}$ dependence of the SHG signals in the SPresonance were also resolved by the analyzer (Fig. 3(b)). The curve is reproduced with a function of $\cos ^{2} \gamma_{\mathrm{a}}$. The evidence indicated that the SHG signals were $p$-polarized. In general, second-order nonlinear susceptibility of the host-guest type NLO polymers can be expressed with the linear combination of the three independent tensor components, that is, $d_{33}, d_{31}\left(=d_{32}\right)$ and $d_{24}\left(=d_{15}\right)$. The present $\gamma_{\mathrm{p}}$ and $\gamma_{\mathrm{a}}$ dependences claim that the present SHG signals are related only to the $d_{33}$ component. The $p$-polarized components of the pump lights excite the SP waves oscillating along the normal of the Ag surface. Subsequently, the SPenhanced fields preferably excite the nonlinear polarizations in the NLO polymers along the normal of the Ag surface.

Finally, Fig. 4 shows the intensity autocorrelation (IAC) trance of the SHG signals of the polymer-coated Ag film in SP-resonance. Again, the thickness of the polymer layers was $15 \mathrm{~nm}$. Here, a pair of the pump pulses propagateting collinearly to the sample surfaces. The timing of the two pulses is controlled with the translational stage driven by a stepping motor. The IAC trace was reproduced with a Gaussian function with a correlation time of $230 \mathrm{fs}$. The IAC trace was also measured in a BBO crystal with $0.1 \mathrm{~mm}$ thickness, and the correlation time was $210 \mathrm{fs}$, corresponding to the pulse width of $150 \mathrm{fs}$. The correlation time of the polymer-coated Ag films is in a good agreement with that in the BBO. Hence, it is concluded that the response time of the SP-enhanced nonlinear susceptibility in the NLO polymers is much shorter than $150 \mathrm{fs}$, the pulse width of the present pump beams.

\section{Conclusions}

In the presentation, we study the second-order nonlinear optical susceptibility of the NLO polymer coated Ag films in the SP-resonance condition. Our present experimental result proved that the surface nonlinearity of the Ag films in the SP-resonance can be effectively improved by coating the NLO polymers. Furthermore, the SP-enhanced nonlinear polarizations in the polymers exhibited the ultrafast response and its responsnce time was much shorter than $150 \mathrm{fs}$.

\section{References}

1. S. E. Irvine, A. Dechant, and A.Y. Elezzabi, "Generation of 0.4-keV Femtosecond Electron Pulses using Impulsively Excited Surface Plasmons" Phys. Rev. Lett. 98, 184801 (2004).

2. S. Kim1, J. Jin, Y.-J. Kim, I.-Y. Park, Y. Kim and S.-W. Kim, "High-harmonic generation by resonant plasmon field enhancement", Nature, 453, 757 (2008).

3. A. Sugita, M. Morimoto, Y. Tamaki, N. Mase, Y. Kawata, S. Tasaka, "Self-organizing secondorder nonlinear susceptibility in NLO-chromophore doped amorphous ferroelectric polymers, poly (cyanophenylene sulfide)", Optical Material Express 2, 1588 (2012). 